\title{
Factors affecting the digestibility of raw and gelatinized potato starches.
}

\begin{abstract}
The enzymatic digestibilities of raw and gelatinized starches in various potato starches, as well as sweet potato, cassava, and yam starches, were estimated, along with other starch properties, such as the phosphorus content, median granule size, and rapid visco analyzer (RVA) pasting properties. Furthermore, correlation coefficients were calculated between the hydrolysis rates (HR) by amylase and other starch quality parameters. A larger granule size was closely associated with a lower HR in raw starch, while the HR in gelatinized starch did not correlate with the median granule size. An increase in phosphorus content resulted in a definitely lower HR in raw starch and tended to decrease the HR in gelatinized starch for the composite of potato and other starches. In contrast, no correlation coefficients of the phosphorus content with the HRs in raw and gelatinized starches were observed within potato starches. Starches with higher peak viscosity and breakdown showed a lower HR in raw starch, while few or no effects of these RVA parameters on the HR in gelatinized starch were observed for the composite of potato and other starches or among potato starches, respectively.
\end{abstract}

Keyword: Potato starch; Digestibility; Phosphorus content; Granule size; Pasting properties. 